Revista científica, arbitrada e indizada, bajo la modalidad electrónica.

Recibido: $15 / 06 / 2020$

Aceptado: 16/07/2020

\title{
DIDÁCTICA TRANSMODERNA DE LA QUÍMICA EN EL NIVEL UNIVERSITARIO
}

Transmodern didactic of chemistry at the university level

\author{
Ramón Antonio Sánchez Rosario \\ rs6869@unphu.edu.do \\ Universidad Bicentenaria de Aragua \\ Venezuela
}

\section{RESUMEN}

El área de Química contribuye al desarrollo integral de los estudiantes, ya que promueve el desarrollo de actitudes y hábitos intelectuales de gran valor en la sociedad actual. Asimismo, facilita la comprensión de fenómenos que tienen lugar en el entorno, ayuda a interpretar de forma racional la realidad y promueve actitudes críticas frente a hechos cotidianos. Por esto, el objetivo del presente estudio es proponer una didáctica transmoderna de la química que favorezca la inclusión de tecnologías y la mezcolanza de las distintas técnicas funcionales empleadas en la actualidad, dentro de estas ABP, aprendizaje basado en proyectos, gamificación, entre otras. En cuanto a la metodología, se efectuó una revisión de literatura sobre la temática con la técnica de matrices de análisis, en conjunto al método hermenéutico y a la experiencia docente. Se concluye con la gestación de una didáctica transmoderna de la Química que permite potenciar la construcción de conocimientos desde los saberes individuales hasta los colectivos, como el conocimiento estratégico, condicional, autoconocimiento y conocimiento afectivo.

Palabras clave: Didáctica, transmoderna, aprendizaje, química.

\begin{abstract}
The Chemistry area contributes to the integral development of students, since it promotes the development of attitudes and intellectual habits of great value in today's society. Likewise, it facilitates the understanding of phenomena that take place in our environment, helps to rationally interpret reality and promotes critical attitudes towards everyday events. For this reason, the objective of this study is to propose a transmodern didactics of chemistry that favors the inclusion of technologies and the hodgepodge of the different
\end{abstract}




\section{Revista científica, arbitrada e indizada, bajo la modalidad electrónica.}

functional techniques currently used, within these PBL, Project-based learning and gamification, among others. Regarding the methodology, a literature review on the subject was carried out with the analysis matrix technique, together with the hermeneutical method and the teaching experience, it concludes with the gestation of a transmodern didactics of Chemistry that allows to enhance the construction of knowledge from individual to collective knowledge, such as strategic, conditional, self-knowledge and affective knowledge.

Keywords: Didactic, transmodern, learning, chemistry

\section{INTRODUCCIÓN}

Alcanzar un aprendizaje significativo en los estudiantes de química permanece siendo un reto para los educadores en todos los niveles. Por medio de la práctica docente a nivel universitario, se puede contemplar como periodo tras periodo los estudiantes de los módulos de química (general, orgánica y analítica), indistinto a sus áreas de concentración se embrollan en la asimilación de información, así como en la construcción de saberes reveladores que puedan extrapolar en sus respectivas disciplinas académicas.

En diversas cátedras de química de la Universidad Nacional Pedro Henríquez Ureña (UNPHU), dentro de estas: química orgánica y análisis instrumental; se ha podido observar disminución en la productividad académica de los estudiantes, que se traduce en asimilación insatisfactoria de los conceptos y en calificaciones insuficientes para aprobar el curso.

De hecho, luego de varias tertulias entre catedráticos de nivel avanzado de la universidad, se concluye que uno de los elementos con mayor peso en este fenómeno es la deficiencia de competencias o experiencias de lecciones pasadas. Sin embargo, los educadores de niveles básicos alegan que tienen la misma situación en sus módulos; sugiriendo que la médula de esta cuestión no está en los docentes, estudiantes, tampoco en las cátedras, sino, en los métodos empleados para conducirlas.

En otras palabras, se deben justipreciar las tácticas de enseñanza empleadas para esta trascendental disciplina, contra las estrategias actuales que podrían ser aplicadas para dicho 


\section{Revista científica, arbitrada e indizada, bajo la modalidad electrónica.}

propósito y establecer una nueva metodología más apropiada para la población estudiantil en cuestión.

El objetivo de este artículo es proponer una metodología transmoderna para el aprendizaje de química, que toma sentido a favor de una reflexión de la valoración del individuo y de un proceso cíclico del aprendizaje. Para ello, la metodología utilizada fue la revisión literaria de diferentes fuentes entre artículos, libros impresos y digitalizados. Así como la sistematización de experiencias del autor. La investigación permitió construir una metodología transmoderna que permite abordar al conocimiento desde distintas vertientes con el objetivo de alcanzar una educación personalizada enfocada en los entes participantes. En la primera parte de este trabajo se explora la enseñanza en la actualidad, algunos de los métodos y técnicas más utilizadas. Luego, se esbozan los conceptos de transmodernidad siguiendo los pensamientos de Rosa María Rodríguez. Además, se atomiza la idea de "educación" desde distintos enfoques; por último, se ofrece una didáctica para el proceso de enseñanza-aprendizaje en química contemplando a los involucrados, cavilando distintas estrategias para la metacognición.

\section{UNA MIRADA A LA ACTUALIDAD DEL APRENDIZAJE DE LA QUÍMICA}

La metacognición es definida por Valenzuela (2019), como un concepto enfocado en el que el proceso de pensamiento se centra en el individuo mismo y la relevancia de este, incrementa en situaciones de aprendizaje. Por otro lado, Bernal et al (2019), relacionan la metacognición con el pensamiento crítico (PC) concluyendo:

Es el proceso del pensamiento que controla y monitorea la ejecución de las habilidades cognitivas de análisis, inferencia y evaluación asociadas al PC; generando una relación bidireccional entre estos dos aspectos ya que el PC es el proceso metacognitivo que mejora el conocimiento a través de la interpretación de las experiencias cognitivas. (p. 213).

En el escrito "una posible definición de metacognición para la enseñanza de las ciencias", Perez y Gonzales (2020), finalizan recalcando la importancia de promover la metacognición en las clases de ciencia y expresan: 


\section{Revista científica, arbitrada e indizada, bajo la modalidad electrónica.}

La mayoría de los sujetos ignora como funciona su mente, por lo que no es capaz de controlarla, (...). Si desde la enseñanza de las ciencias comenzamos a desarrollar en los estudiantes la capacidad de conocer cómo funciona su cognición, y habilitamos espacios para controlarla, estaremos brindando herramientas para que se defiendan de los discursos hegemónicos en los que viven inmersos. (p. 398).

El modelo sustentado en la transmisión de conocimientos desde el catedrático del quehacer profesoral continúa siendo ampliamente utilizado en la práctica docente de química. Un paradigma que naufraga en responder a las exigencias de una sociedad cambiante, transdisciplinaria y transmoderna.

Sin embargo, los modelos actuales exigen un cambio en los actores tradicionales docente-estudiante. Dichos modelos, tienen como cúspide la construcción del conocimiento por parte de los educando en un concepto que se denomina "aprender a aprender" (AaA).

Caena y Stringher (2020) definen el AaA como: "una compleja hiper-competencia que orquesta recursos cognitivos, metacognitivos y socio-afectivo-motivacionales del individuo, en un esfuerzo voluntario para producir conocimiento y mejora sobre el propio aprendizaje” (p. 206). Exponen también, que a medida que el individuo avanza en edad, el AaA "se convierte en una compleja mezcla de habilidades, conocimientos y comportamientos que abarcan recursos cognitivos, metacognitivos y socio-afectivomotivacionales".

Galagovsky (2009), explica que la complejidad del lenguaje utilizado en los discursos pedagógicos de química es un componente significativo en la incomprensión de los conceptos. Ramírez y Mosquera (2018), indican "El lenguaje no solo transmite, el lenguaje crea o constituye el conocimiento o la 'realidad". En cambio, Caamaño y Onorbe (2004), exponen que más allá de los aspectos conceptuales, “es necesario replantearse los objetivos, los contenidos y las estrategias didácticas de la química", también arguyen que:

Estos cambios deberían ir acompañado de un mayor conocimiento sobre las dificultades de aprendizaje de los contenidos de la química y de un uso más eficaz de las estrategias didácticas y de los recursos disponibles para ayudar a superarlas, para conseguir de este modo una mejor 


\section{Revista científica, arbitrada e indizada, bajo la modalidad electrónica.}

comprensión de los principios básicos de la química, de sus aplicaciones, y de su relación con otras ciencias y con la sociedad. (p.79).

Por consiguiente, múltiples estrategias didácticas transmodernas alineadas a la transdisciplinariedad han sido aprovechadas en la enseñanza de química y sus resultados confrontados. Por ejemplo, Sandoval et al. (2013), contrastaron: tutorías docentes, Aprendizaje Basado en Problemas (ABP), visitas educativas extraclases, experimentación en aula/laboratorio, problemas integradores de conocimientos y problemas domiciliarios grupales. En este trabajo, se concluye que "todas las propuestas metodológicas mencionadas son claramente transferibles al desarrollo de contenidos de las cátedras de Química y del resto de las asignaturas de las carreras de nuestra Universidad y de otras instituciones de educación superior" (p.136). De igual importancia, aspectos como cambio de percepción hacia la química, mejoramiento de interacción entre compañeros y con el docente, instauración de lazos sociales también fueron observados con las distintas metodologías implementadas.

Por otra parte, Nakamatsu (2012), manifiesta que el empleo de la secuencia demostración-explicación capta la atención del estudiante y adicional a esto, promueven la observación y el descubrimiento de cambios.

En la actualidad, estrategias como ABP (Cano y Gideeg, 2017), aprendizaje basado en simulaciones (Plass, 2012), y aprendizaje basado en simulaciones e integración de videos (Kestin, 2020), gamificación (Sousa et al., 2019), aprendizaje basado en proyectos (Davis et al, 2017), aprendizaje basado en proyectos con mediación de las TIC (RomeroValderrama et al., 2018), se emplean con mayor frecuencia cada día en busca de la herramienta apropiada para la enseñanza de la química.

\section{TRANSMODERNIDAD Y EDUCACIÓN}

A partir de la revisión de los acercamientos conceptuales de diversos teóricos, se comprende la importancia de las concepciones de los "post" del siglo XX para revalorizar el prefijo "trans" del siglo XXI, que es el fundamento transgresor de muchas de las relaciones complejas que se viven en la actualidad. La filósofa española, Rodríguez Magda, sitúa la actualidad como una época transmoderna. La misma autora refiere que la 


\section{Revista científica, arbitrada e indizada, bajo la modalidad electrónica.}

comprensión de la condición presente, que la actualidad no se caracteriza por la ruptura, por tanto no se enmarca en el prefijo "post" de fin de siglo, sino en el "trans" de comienzos de milenio: Rodríguez (2004), afirma que "el prefijo que debe guiar la nueva razón digital en una realidad virtual y fluctuante"(p.30).

Para Rodríguez (2004), “Trans” es el prefijo que se iguala con lo transcultural, la transmisibilidad, la transexualidad, la transformación, lo transnacional, lo transgénico, la transgresión y significativamente con la transformación y la transcendencia: “"Trans' es transformación, dinamismo, atravesamiento de algo en un medio diferente: es una tendencia a la razón digital de la existencia; ese algo que va 'a través de', no se estanca, sino que parece alcanzar un estadio posterior, conlleva por tanto la noción de transcendencia" (ibíd.: 16, Citado por Ayala, 2016, p. 65).

La transmisibilidad se relaciona con la capacidad del alumno de reconocer su propio aprendizaje y hacerlo consciente a través de lo que llamamos "reflexiones de conocimiento" en donde expone los diferentes grados de metacognición; es decir, la transmisibilidad se observa en el trasvase de la actividad, proyecto o trabajo realizado para materializar su aprendizaje en una reflexión continua de su propio proceso de conocimiento

El término transmodernidad es descrito por Rodríguez Magda (2011), como la trascendencia de la modernidad:

La Transmodernidad prolonga, continúa y transciende la Modernidad, es el retorno, la copia, la pervivencia de una Modernidad débil, rebajada, light. La zona contemporánea transitada por todas las tendencias, los recuerdos, las posibilidades; transcendente y aparencial a la vez, voluntariamente sincrética en su "multicronía". Un retorno, distanciado, irónico, que acepta su ficción útil. La Transmodernidad es lo postmoderno sin su inocente rupturismo, es imagen, serie, barroco de fuga y autorreferencia, catástrofe, bucle, reiteración fractal e inane; entropía de lo obeso, inflación amoratada de datos; estética de lo repleto y de su desaparición, entrópica, fatal. Su clave no es el post, la ruptura, sino la transubstanciación vasocomunicada de los paradigmas. La Transmodernidad no es un deseo o una meta, simplemente está, como una situación estratégica, compleja y aleatoria no elegible; no es buena ni mala, benéfica o insoportable... y es todo eso juntamente... Es el 


\section{Revista científica, arbitrada e indizada, bajo la modalidad electrónica.}

abandono de la representación, el reino de la simulación, de la simulación que se sabe real" (p.6).

Por otra parte, Dussel discurre que la transmodernidad debe apropiarse de la revolución tecnológica moderna, desechando lo antiecológico, exponiéndola al servicio de mundos antiguos con tradiciones propias y creatividad ignorada. Logrando una riqueza cultural y humana garrafal que intenta suprimir el mercado capitalista transnacional. El autor finiquita indicando que "es el retorno a la conciencia de las grandes mayorías de la humanidad de su inconsciente histórico excluido” (Dube y Mignolo, 2004).

Partiendo de los asertos anteriores, Aldo Ahumada Infante concluye que "la Transmodernidad se propone ver desde el otro los fenómenos y discursos hegemónicos que se producen y se enuncian desde el centro" (Infante, 2013). Esto es, vislumbrar con crítica postmoderna lo planteado por el modernismo, evaluar el esbozo eurocéntrico catando las realidades de los marginados.

Durkheim (como se citó en Padilla, 2006) piensa que la educación:

La educación es la acción ejercida por las generaciones adultas que no han alcanzado todavía el grado de madurez necesario para la vida social. Tiene por objeto el suscitar y desarrollar en el niño un cierto número de estados físicos, intelectuales y morales que exigen de él tanto la sociedad política en su conjunto como el medio ambiente especifico al que está especialmente destinado. (p.115)

Durkheim supone que la integración, adaptación y selección de talentos encargados de gestionar la sociedad industrial, se debe a la escuela. Considerando a la educación como subsistema social equilibrado y funcional que cubre las demandas de la sociedad industrial (Moreno, 2001).

La educación de calidad, según Ugas (2006), “es la que ofrece contenidos que el individuo necesita como persona (intelectual, afectiva y moral) para desempeñarse adecuadamente en los diferentes ámbitos de la sociedad”. La calidad de la enseñanza no radica según lo expresado por el autor en la transmisión de los contenidos, sino en la comprensión que el educando tenga de los mismos. Es allí donde la gestión del docente 


\section{Revista científica, arbitrada e indizada, bajo la modalidad electrónica.}

debe centrar su labor en hacer un proceso productivo de enseñanza aprendizaje que propicie la formación integral del estudiante.

Ramírez y Mosquera (2018), esbozan “la educación es un proceso de transformación la convivencia, a través del cual el ser que aprende se conserva en su humanidad o se pierde en el devenir de la historia, a partir de su formación” (p.100). A su vez, comentan:

Ser un profesor del siglo XXI es dejar sus viejas ideas y creencias de lado y asumir su verdadero rol, partiendo del principio que todo ser humano hace parte de una sociedad y que no solo se educa en la escuela, (...).No podemos concebir el futuro de las nuevas generaciones sin pensar que existe más de una verdad, que lo científico no es lo único y que el ser humano no es un conjunto de partes aisladas. (p.103).

\section{METODOLOGÍA}

El presente artículo está fundamentado en la interpretación bibliográfica de libros y artículos de revistas y el método cualitativo hermenéutico. Es importante la selección de los autores a revisar, con la finalidad de direccionar y reducir el tiempo de búsqueda.

Gómez-Luna (2014) recomienda:

La aplicación de criterios de selectividad de manera que el investigador se enfoque en los documentos relevantes para la investigación y si, a pesar de todo, existe demasiada información, quizás el tema elegido sea excesivamente amplio y sea necesario enfocarlo aún más. (p.160).

Con la finalidad de direccionar y reducir el tiempo de búsqueda. Así mismo, los métodos cualitativos "posibilitan recopilar las percepciones, perspectivas y voces de los investigados" (Iño, 2018). Por otra parte, Ruedas (2008), expone que:

La actividad hermenéutica hace posible el proceso de adquisición de saberes y de interpretación, por lo que constituye la base de una personalidad crítica y reflexiva; en consecuencia, otorga al individuo la capacidad de percibir "literalmente" la realidad para poder implementar los cambios que considere necesarios en pro del bienestar individual y colectivo. (p.196). 


\section{Revista científica, arbitrada e indizada, bajo la modalidad electrónica.}

\section{RESULTADOS}

Para cumplir con el objetivo propuesto, gestar la didáctica transmoderna para la enseñanza de química, se confrontaron por medio de la contrastación, la información encontrada en la revisión bibliográfica y la información obtenida de la experiencia docente.

Una Didáctica Transmoderna de la Química desde la triada docenteestudiante-metacognición.

De las evidencias anteriores, se identifican elementos que combinados podrían moldear una didáctica consonante con las necesidades y deficiencias identificadas por los distintos autores y docentes. Las ideas y reflexiones expuestas fusionadas con las experiencias del autor, gestan la metodología "Química viable", que se describe a continuación:

La química viable (QV) se fundamenta en la mezcolanza de la búsqueda de información tradicional, sumado al empleo de softwares, simuladores y aplicaciones interactivas que acerquen los conceptos al entendimiento de los estudiantes. En esta, el docente señala los temas a desarrollar en las sesiones de clase, empleando libros, revistas o internet. Luego, surge una discusión de lo encontrado con la finalidad de validar los conceptos. Una vez homologado, se utilizan softwares, simuladores de ordenador o aplicaciones móviles. Los estudiantes comprueban sus conocimientos de una manera recreativa $\mathrm{y}$, tienen la oportunidad de interpretar ideas abstractas poco asimilables con la mera lectura o dilucidación del profesor. Numerosas aplicaciones disponibles cuentan con juegos que permiten acumular puntos, asistiendo al profesor en seguimiento a la construcción de los conocimientos y su vez, estimulando al estudiante a experimentar en casa.

Existen aplicaciones para todas las áreas de la química. Para el presente escrito se emplearon los siguientes: 


\section{Revista científica, arbitrada e indizada, bajo la modalidad electrónica.}

\begin{tabular}{|c|c|c|c|}
\hline Nombre App & $\begin{array}{l}\text { Área de } \\
\text { Química }\end{array}$ & Descripción & $\begin{array}{l}\text { Desarrollado } \\
\text { por }\end{array}$ \\
\hline $\begin{array}{l}\text { Analytical } \\
\text { Chemistry } \\
\text { Challenge }\end{array}$ & Analítica & $\begin{array}{l}\text { Proporciona pruebas de selección múltiple para } \\
\text { asentar los conocimientos adquiridos. }\end{array}$ & $\begin{array}{l}\text { Departamento de } \\
\text { química HKBU }\end{array}$ \\
\hline Chemistry Lab & Orgánica & $\begin{array}{l}\text { Poderosa herramienta que ofrece pruebas de selección } \\
\text { múltiple para la validación de conceptos. Además, } \\
\text { contiene ejercicios para adiestrar a los usuarios con } \\
\text { los mecanismos de reacciones orgánicas, dotándolos } \\
\text { de competencias claves en esta área de la química. }\end{array}$ & $\begin{array}{l}\text { Electrolytic } \\
\text { Earth }\end{array}$ \\
\hline $\begin{array}{l}\text { Virtual Orbitals - } \\
\text { 3D }\end{array}$ & $\begin{array}{l}\text { General/ } \\
\text { Orgánica }\end{array}$ & $\begin{array}{l}\text { Este simulador ofrece los conceptos de la teoría de } \\
\text { orbitales de una manera clara y precisa. Ofrece la } \\
\text { oportunidad de jugar con los orbitales en sus distintas } \\
\text { orientaciones, ofreciendo ejemplos de elementos y } \\
\text { compuestos prediseñados. }\end{array}$ & Anurag Anand \\
\hline $\begin{array}{c}\text { PhET - } \\
\text { Interactive } \\
\text { Simulations (Ley } \\
\text { de Beer Lambert) }\end{array}$ & Analítica & $\begin{array}{l}\text { Es un simulador que permite experimentar como } \\
\text { influye la concentración de una disolución con la } \\
\text { medida de absorbancia. }\end{array}$ & $\begin{array}{l}\text { University of } \\
\text { Colorado } \\
\text { Boulder }\end{array}$ \\
\hline $\begin{array}{c}\text { Solution } \\
\text { Calculator Lite } \\
\end{array}$ & $\begin{array}{l}\text { General/ } \\
\text { Analítica }\end{array}$ & $\begin{array}{l}\text { Brinda una calculadora rápida, precisa y fácil de usar } \\
\text { para preparar disoluciones químicas }\end{array}$ & Cooloy \\
\hline Química Analítica & Analítica & $\begin{array}{l}\text { Proporciona definiciones claras sobre los términos } \\
\text { asociados con esta rama de la química }\end{array}$ & Kirill Sidorov \\
\hline
\end{tabular}

Figura No.1: Aplicaciones disponibles en App Store de la plataforma Android.

\section{DISCUSIÓN}

Las investigaciones sobre las distintas técnicas que se emplean en la enseñanza de la química sostienen que, se deben considerar los aspectos sociales incidentes sobre el individuo, de manera que los distintos 'backgrounds' de los estudiantes no afecten el resultado esperado de la didáctica implementada. Asimismo, la inclusión en el proceso enseñanza-aprendizaje de actividades que permitan la ejecución y experimentación de estas con poca interacción del docente, favorecería el estado de motivación del participante, propiciando las condiciones ideales para un aprendizaje significativo.

Por otro lado, la incorporación de las aplicaciones móviles y juegos disponibles en las plataformas digitales en el proceso educativo, es un aliado significativo al momento de diseccionar conceptos abstractos presentes en asignaturas de ciencias como química y física. De hecho, incluir los programas informáticos 'Virtual Orbitals-3D' y 'Chemistry $L a b$ ' en la cátedra de estereoquímica de química orgánica mejoró de manera considerable 


\section{Revista científica, arbitrada e indizada, bajo la modalidad electrónica.}

las evaluaciones de los estudiantes, pues en sus palabras, "finalmente entendieron como se ve un orbital atómico".

\section{CONCLUSIONES}

El manejo de aplicaciones informáticas (Apps) como parte de las estrategias de enseñanza de la química facilita la compresión de ideas abstractas, presentes con frecuencia, en esta área del saber científico. De igual manera, suscita entusiasmo en los estudiantes para su utilización.

Los recursos económicos no implican una limitación para el empleo de esta estrategia, puesto que, las universidades cuentan con áreas con ordenadores disponibles para la población estudiantil y profesoral, y en algunos casos, para el público en general. Otro aspecto a considerar es que las relaciones interpersonales resultan favorecidas con la aplicación de esta didáctica transmoderna; ciertas aplicaciones ofrecen la opción de trabajar en equipos, como lo es “The Open Drug Discovery Teams (ODDT)” (Sean Ekins, 2012).

La figura del profesor en esta propuesta didáctica es la del experto consultor. Semejante al ABP, el profesor debe certificar que los estudiantes van por la vía correcta durante la primera parte de la estrategia y con ayuda de las Apps reafirmar que el aprendizaje es significativo. La utilización de la QV incide en la capacidad metacognitiva de los estudiantes, puesto que, permite la realización de los pensamientos durante la solución de problema, y, permite conocer que conoce el individuo (como se cita en Vargas y Arbeláez, 2002). La estrategia propuesta apoyada en el uso de las TIC facilitaría la creación de comunidades de colaboración continua y permanente (Colina y Tua, 2018). Se recomienda la realización de estudios posteriores implementando el aprendizaje basado en retos a la par con la estrategia $\mathrm{QV}$, que promete ser una aliada vanguardista y efectiva en la transmisión de saberes en el área de química (Olivares et al., 2018).

\section{REFERENCIAS BIBLIOGRÁFICAS}

Ayala, J. J. (2016). El fundamento "trans" para la didáctica transmoderna. CAUCE Revista Internacional de Filología, Comunicación y sus Didácticas(39). 
Revista científica, arbitrada e indizada, bajo la modalidad electrónica.

Bernal, M.E., Gómez, M. e Iodice, R. (2019). Interacción conceptual entre el pensamiento crítico y metacognición. Revista Latinoamericana de Estudios Educativos, 15 (1), 193-217

Caena, F., \& Stringher, C. (2020). Hacia una nueva conceptualización del Aprender a Aprender. Aula abierta. 49(3), 199-216.

Cano, M. F. C., y Gideeq, G. (2018). Problem Based Learning (PBL), competencies and Chemistry teaching for Engineers.

Colina, A. M., \& Tua, J. J. (2018). Apropiación de las TIC en la docencia universitaria: Statu quo ante un panorama complejo. Revista Espacios, 39(43).

Davis, E. J., Pauls, S., \& Dick, J. (2017). Project-based learning in undergraduate environmental chemistry laboratory: using EPA methods to guide student method development for pesticide quantitation. Journal of Chemical Education, 94(4), 451-457.

Dube, S., Banerjee Dube, I., \& Mignolo, W. (2004). Modernidades coloniales: otros pasados, historias presentes. México: El Colegio de México, Centro de estudios de Asia y África .

Galagovsky, L. (2009). Enseñanza de la química: lenguajes expertos como obstáculos de aprendizaje. Enseñanza de las Ciencias, Número Extra VIII Congreso Internacional sobre Investigación en Didáctica de las Ciencias, Barcelona, pp. 425429. http://ensciencias.uab.es/congreso09/numeroextra/art-425-429.pdf

Gómez-Luna, E., y Fernando-Navas, D., y Aponte-Mayor, G., y Betancourt-Buitrago, L. A. (2014). Metodología para la revisión bibliográfica y la gestión de información de temas científicos, a través de su estructuración y sistematización. Dyna, 81(184), undefined-undefined. [fecha de Consulta 3 de Noviembre de 2019]. ISSN: 0012-7353. Disponible en: https://www.redalyc.org/articulo.oa?id=496/49630405022

Infante, A. A. (2013). Transmodernidad: dos proyectos disímiles bajo un mismo concepto. Polis, Revista Latinoamericana, 12(34), 291-305.

Iño Daza, W. (2018). Investigación educativa desde un enfoque cualitativo: la historia oral como método. Voces De La Educación, 3(6), 93 - 110. Recuperado a partir de https://www.revista.vocesdelaeducacion.com.mx/index.php/voces/article/view/123

Kestin, G., Miller, K., McCarty, L. S., Callaghan, K., \& Deslauriers, L. (2020). Comparing the effectiveness of online versus live lecture demonstrations. Physical Review Physics Education Research, 16(1), 013101

Moreno, A. (2001). Sociología de la Educación: Claves Teóricas. Asturias: Septem Ediciones.

Nakamatsu, J. (2012). Reflexiones sobre la enseñanza de la Química. En Blanco \& Negro, 3(2), 38-46.

Olivares, S. L. O., Cabrera, M. V. L., \& Valdez-García, J. E. (2018). Aprendizaje basado en retos: una experiencia de innovación para enfrentar problemas de salud pública. Educación Médica, 19, 230-237. 
Revista científica, arbitrada e indizada, bajo la modalidad electrónica.

Onorbe, A. C. (2004). La enseñanza de la química: concepto y teorías, dificultades de aprendizaje y replanteamiento curriculares. $68-81$.

Padilla Pineda, M. T. (2006). Émile Durkheim: Estructura social y subjetividad. UAEH.

Pérez, G., \& Galli, L. M. G. (2020). una posible definición de metacognición para la enseñanza de las ciencias. Investigacões Em Ensino De Ciências, 25(1), 385-404. doi:http://dx.doi.org/10.22600/1518-8795.ienci2020v25n1p384

Piastro, E. (1996). Para qué educar. En: Revista Didac $\mathrm{N}^{\mathrm{o}} 33$. Universidad Iberoamericana.

Plass, J. L., Milne, C., Homer, B. D., Schwartz, R. N., Hayward, E. O., Jordan, T., ... y Barrientos, J. (2012). Investigating the effectiveness of computer simulations for chemistry learning. Journal of Research in Science Teaching, 49(3), 394-419.

Ramírez Mattiazzi, Leonor y Mosquera Liliana. (2018). Educar o enseñar en el Siglo XXI. Retos de la Ciencia, 2(2), pp. 98-104.

Romero-Valderrama, A. C., Forero-Romero, A., y Rodríguez-Hernández, A. A., (2018). Análisis comparación del aprendizaje basado en proyectos de forma tradicional y con mediación de las TIC. Revista ESPACIOS, 39(52)

Rodríguez Magda, R. M. (2004). Transmodernidad. Barcelona: Anthropos Editorial.

Rodríguez Magda, R. M (2011). Transmodernidad: un nuevo paradigma. Transmodernity, 1(1), 1-13.

Ruedas Marrero, M., Ríos Cabrera, M. M., y Nieves, F. (2009). Hermenéutica: la roca que rompe el espejo. Investigación y Postgrado, 24(2), undefined-undefined. [fecha de Consulta 3 de Noviembre de 2019]. ISSN: 1316-0087. Disponible en: https:/www.redalyc.org/articulo.oa?id=658/65817287009

Sandoval, Marisa Julia, y Mandolesi, María Ester, y Cura, Rafael Omar (2013). Estrategias didácticas para la enseñanza de la química en la educación superior. Educación y Educadores, 16(1),126-138.[fecha de Consulta 24 de Noviembre de 2020]. ISSN: 0123-1294. Disponible en: https://www.redalyc.org/articulo.oa?id=834/83428614007

Sean Ekins, A. M. (2012). Open Drug Discovery Teams: A chemistry Mobile App for Collaboration. Molecular informatics, 585-597.

Sousa Lima, M. A., Monteiro, Á. C., Melo Leite Junior, A. J., de Andrade Matos, I. S., Alexandre, F. S. O., Nobre, D. J., ... y da Silva Júnior, J. N. (2019). Game-based application for helping students review chemical nomenclature in a fun way.

Ugas, G. (2006). La Complejidad. Un modo de pensar. Ediciones del Taller Permanente de Estudios Epistemológicos en Ciencias Sociales. Táchira Venezuela.

Valenzuela, Á. (2019). ¿Qué hay de nuevo en la metacognición? Revisión del concepto, sus componentes y términos afines. Educação e Pesquisa, 45, e187571-e187571.

Vargas, E. y Arbeláez, M. (2002). Consideraciones teóricas acerca de la metacognición. Revista de Ciencias Humanas UTP, 28, 161-170. Universidad Tecnológica de Pereira. 